\title{
Management of Severe COVID-19 Infection in Children: A Therapeutic Challenge
}

\author{
Itzhak Brook
}

Although coronavirus disease 2019 (COVID-19) occurs in children, compared to adults they have a milder disease. They are infrequently admitted to intensive care units, have better prognosis and their mortality is low [1]. However, because children are often asymptomatic or experience a mild disease the true incidence of COVID-19 in children may be higher. Infected children should be isolated and mild cases should receive supportive treatment at home [2]. The use of antiviral or immunomodulatory therapies should only be considered within a clinical trial setting or on a case-to-case basis [2].

Pediatricians should be watchful for the uncommon but serious post-infectious Kawasaki-like, pediatric multisystem inflammatory syndrome related to COVID-19 which may occur several weeks following an asymptomatic or mild infection [2].

Children with serious manifestation of COVID-19, such as septic shock, altered consciousness or multi-organ failure, severe acute respiratory distress syndrome, pediatric multisystem inflammatory syndrome, should be admitted to the hospital and receive supportive care, and organ support in case of organ failure. Administration of antiviral and immunomodulatory therapy may be required.

To achieve maximal effect, antivirals should be administered as early as possible before clinical deterioration occurs [3]. Therapeutic options include lopinavir/ritonavir and ribavirin for 7 days, and remdesivir for 10 days [4]. Clinical trials of chloroquine and hydroxychloroquine in adults with $\mathrm{COV}$ ID-19 infection have shown no efficacy [5].

Some children with acute respiratory distress syndrome manifest clinical features and serological markers seen in hyper inflammatory syndromes. The levels of these markers are often lower than those seen in other syndromes [6]. These markers include chimeric antigen receptor $\mathrm{T}$ cell therapy-associated cytokine release syndrome, secondary hemophagocytic lymphohistiocytosis, and sepsis-associated macrophage activationlike syndrome. Most of the inflammation in COVID-19 occurs within the lungs [7]. Children with multisystem inflammatory syndrome should be treated with immunomodulatory therapy.

The decision to initiate antiviral and immunomodulatory therapy for COVID-19 should be made after carefully consid-

Manuscript submitted August 29, 2020, accepted September 2, 2020

Published online November 3, 2020

Department of Pediatrics, Georgetown University School of Medicine, Washington, DC 20016, USA. Email: dribrook@yahoo.com eration on individual basis. This is because there is currently no proof of their effectiveness for COVID-19 in children and only limited clinical evidence in adults $[8,9]$.

Therapeutic choices include humanized anti- interleukin 6 (IL-6) monoclonal antibody (tocilizumab) [10], and a recombinant antagonist of the human IL-1 receptor (anakinra) [11].

Corticosteroids may be helpful in the management of rapidly worsening chest imaging and presence of acute respiratory distress syndrome, septic shock, toxic symptoms, encephalitis or encephalopathy, secondary hemophagocytic lymphohistiocytosis, and wheezing $[12,13]$.

Those manifesting pediatric multisystem inflammatory syndrome with clinical features similar to Kawasaki disease should be treated with high-dose intravenous immunoglobulin, corticosteroids (methylprednisolone), aspirin, heparin, and immunomodulatory agents (e.g., tocilizumab and anakinra) [14].

Respiratory support is essential in those with respiratory distress, and includes non-invasive measures such as heated humidified oxygenation administered through nasal cannula or mask, continuous positive airway pressure, or high-frequency ventilation [15]. Mechanical ventilation should be initiated if these measures fail.

Hemofiltration or hemodialysis may be necessary in those with multiple organ failure (especially acute kidney injury) or fluid overload. Plasma exchange is required to treat liver failure [15]. Extracorporeal membrane oxygenation may be needed when mechanical ventilation or hemofiltration fail, and cardiopulmonary failure occurs [16].

More research is needed to better define and understand the disease course and pathophysiology of COVID-19 infection and pediatric multisystem inflammatory syndrome in children. Ongoing clinical trials investigating the use of antiviral and immunomodulatory agents may better define their role in the management of these conditions. Because of the paucity of solid evidence to support the therapeutic choices, the decision to initiate any of these treatments should be made carefully on an individual basis [17].

\section{Acknowledgments}

None to declare.

\section{Financial Disclosure}

None to declare. 


\section{Conflict of Interest}

None to declare.

\section{Data Availability}

The author declares that data supporting the findings of this study are available within the article.

\section{References}

1. Ludvigsson JF. Systematic review of COVID-19 in children shows milder cases and a better prognosis than adults. Acta Paediatr. 2020;109(6):1088-1095.

2. She J, Liu L, Liu W. COVID-19 epidemic: Disease characteristics in children. J Med Virol. 2020;92(7):747-754.

3. Pascarella G, Strumia A, Piliego C, Bruno F, Del Buono R, Costa F, Scarlata S, et al. COVID-19 diagnosis and management: a comprehensive review. J Intern Med. 2020;288(2):192-206.

4. Wiersinga WJ, Rhodes A, Cheng AC, Peacock SJ, Prescott HC. Pathophysiology, transmission, diagnosis, and treatment of coronavirus disease 2019 (COVID-19): A Review. JAMA. 2020.

5. Chowdhury MS, Rathod J, Gernsheimer J. A rapid systematic review of clinical trials utilizing chloroquine and hydroxychloroquine as a treatment for COVID-19. Acad Emerg Med. 2020;27(6):493-504.

6. Phadke M, Saunik S. COVID-19 treatment by repurposing drugs until the vaccine is in sight. Drug Dev Res. 2020;81(5):541-543.

7. Wang D, Hu B, Hu C, Zhu F, Liu X, Zhang J, Wang B, et al. Clinical characteristics of 138 hospitalized patients with 2019 novel coronavirus-infected pneumonia in Wuhan, China. JAMA. 2020.

8. Beigel JH, Tomashek KM, Dodd LE, Mehta AK, Zingman BS, Kalil AC, Hohmann E, et al. Remdesivir for the treat- ment of COVID-19 - preliminary report. N Engl J Med. 2020.

9. Lu X, Zhang L, Du H, Zhang J, Li YY, Qu J, Zhang W, et al. SARS-CoV-2 Infection in Children. N Engl J Med. 2020;382(17):1663-1665.

10. Xu X, Han M, Li T, Sun W, Wang D, Fu B, Zhou Y, et al. Effective treatment of severe COVID-19 patients with tocilizumab. Proc Natl Acad Sci U S A. 2020;117(20):1097010975.

11. Dimopoulos G, de Mast Q, Markou N, Theodorakopoulou M, Komnos A, Mouktaroudi M, Netea MG, et al. Favorable anakinra responses in severe COVID-19 patients with secondary hemophagocytic lymphohistiocytosis. Cell Host Microbe. 2020;28(1):117-123 e111.

12. Jean SS, Lee PI, Hsueh PR. Treatment options for COVID-19: the reality and challenges. J Microbiol Immunol Infect. 2020;53(3):436-443.

13. Ye Z, Wang Y, Colunga-Lozano LE, Prasad M, Tangamornsuksan W, Rochwerg B, Yao L, et al. Efficacy and safety of corticosteroids in COVID-19 based on evidence for COVID-19, other coronavirus infections, influenza, community-acquired pneumonia and acute respiratory distress syndrome: a systematic review and meta-analysis. CMAJ. 2020;192(27):E756-E767.

14. Feldstein LR, Rose EB, Horwitz SM, Collins JP, Newhams MM, Son MBF, Newburger JW, et al. Multisystem Inflammatory Syndrome in U.S. Children and Adolescents. N Engl J Med. 2020;383(4):334-346.

15. Shen KL, Yang YH, Jiang RM, Wang TY, Zhao DC, Jiang $\mathrm{Y}, \mathrm{Lu} \mathrm{XX}$, et al. Updated diagnosis, treatment and prevention of COVID-19 in children: experts' consensus statement (condensed version of the second edition). World J Pediatr. 2020;16(3):232-239.

16. MacLaren G, Fisher D, Brodie D. Preparing for the Most Critically Ill Patients With COVID-19: The Potential Role of Extracorporeal Membrane Oxygenation. JAMA. 2020.

17. Naja M, Wedderburn L, Ciurtin C. COVID-19 infection in children and adolescents. Br J Hosp Med (Lond). 2020;81(8):1-10. 